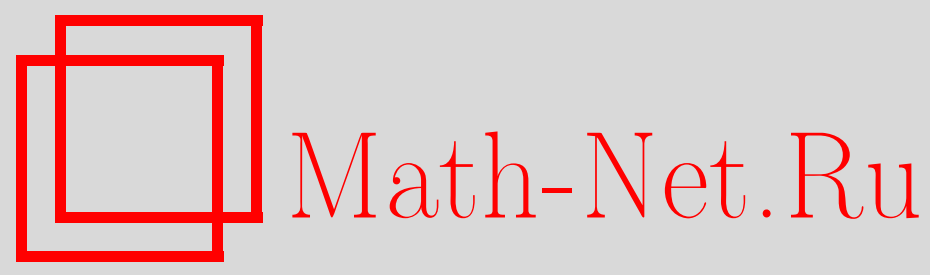

В. В. Горбацевич, О свойствах плезиоравномерных подгрупп в группах Ли, Матем. заметки, 2001, том 69, выпуск $3,338-345$

DOI: https://doi.org/10.4213/mzm507

Использование Общероссийского математического портала Math-Net.Ru подразумевает, что вы прочитали и согласны с пользовательским соглашением http://www.mathnet.ru/rus/agreement

Параметры загрузки:

IP: 52.90 .164 .192

26 апреля 2023 г., 16:54:03

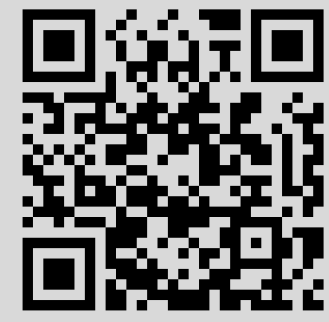




\section{О СВОЙСТВАХ ПЛЕЗИОРАВНОМЕРНЫХ ПОДГРУПП В ГРУППАХ ЛИ}

\section{В.В. Горбацевич}

Работа посвящена изучению свойств одного класса подгрупп $H$ в группах Ли $G$, который был ранее введен автором. Замкнутая подгруппа $H$ в группе Ли $G$ называется плезиоравномерной, если существует некоторая замкнутая подгруппа $P$ в $G$, содержащая $H$ и такая, что $P$ равномерна в $G$, а $H$ квазиравномерна в $P$. В статье даются ответы на несколько естественных вопросов о плезиоравномерных подгруппах. Доказано, что если в определении плезиоравномерности поменять местами равномерность и квазиравномерность подгрупп, получается то же понятие плезиоравномерности. Если замкнутая подгруппа $H$ в $G$ содержит плезиоравномерную подгруппу, то $H$ сама будет плезиоравномерна. Рассмотрены и другие свойства плезиоравномерных подгрупп.

Библиография: 11 названий.

Эта работа посвящена изучению свойств одного класса подгрупп $H$ в группах Ли $G$, который был введен автором в работе [1]. (Здесь и во всей работе под подгруппой группы Ли мы понимаем подгруппу Ли.) Эти подгруппы $H$ изучались там в связи с соответствуюшими однородньми пространствами $G / H$, которые получили название плезиокомпактных однородных пространств. Оказалось, что на плезиокомпактные однородные пространства распространяются многие результаты, которые ранее были получены (в том числе и автором, см., например, обзор [2]) для компактных однородных пространств (см. [1], [3], [4]). В данной же работе мы посмотрим на ситуацию несколько с другой стороны и будем изучать не столько однородные пространства, сколько сами подгрупшы (хотя, конечно, оба эти подхода эквивалентны).

Подгруппа Ли $H$ в групе Ли $G$ назьвается равномерной, если она замкнута в $G$ и однородное пространство $G / H$ компактно. Еще один важньй класс подгрупп в $G$ - это те, для которых однородное пространство $G / H$ имеет конечную инвариантную меру (конечность меры означает, что объем пространства $G / H$ относительно указанной инвариантной меры конечен). Такиеподгрупшы $H$ называют квазиравномернылми, а соот-

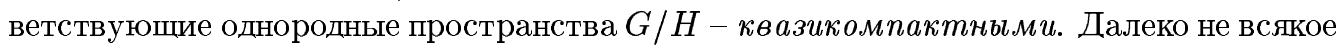
компактное однородное пространство будет квазикомпактно, ибо на нем может просто не оказаться инвариантной меры. Однако любое компактное однородное пространство компактной или разрешимой групшы Ли будет, как хорошо известно, квазикомпактно (для однородных пространств разрешимых групп Ли это утверждение нетривиально и

Работа выполнена при финансовой поддержке Российского фонда фундаментальных исследований, грант № 98-01-00329. 
было доказано в [5]). Понятие плезиоравномерной подгрупшы можно рассматривать как обобщение для обоих понятий - как равномерньх, так и квазиравномерных подгрупп.

Замкнутая подгруппа $H$ в группе Ли $G$ (такая $H$ автоматически является подгруппой Ли в $G$ ) назьвается плезиоравномерной, если существует некоторая замкнутая подгруппа $P=P_{H}$ в $G$, содержащая $H$ и такая, что $P$ равномерна в $G$, а $H$ квазиравномерна в $P$. Ясно, что понятия равномерности и квазиравномерности - частные случаи понятия плезиоравномерности. Есть еще несколько эквивалентных определений плезиоравномерной подгрупшы (см. [1]). Нетрудно привести примеры плезиоравномерных подгруп, которые не являются ни равномерньми, ни квазиравномерными. Например, пусть $G_{1}$ и $G_{2}$ - две группы Ли, а $H_{1}$ и $H_{2}$ - две замкнутые подгруппы в них, причем $H_{1}$ равномерна в $G_{1}$, но $G_{1} / H_{1}$ не имеет конечной инвариантной меры (например, таковы пространства $G / B$, где $B$ - борелевская подгруппа в комплексной полупростой группе Ли $G$ ) и $H_{2}$ квазиравномерна в $G_{2}$, но не равномерна в ней (например, $\mathrm{SL}_{2}(\mathbb{Z})$ в $\left.\mathrm{SL}_{2}(\mathbb{R})\right)$. Тогда, очевидно, подгруппа $H_{1} \times H_{2}-$ плезиоравномерная подгруппа в $G_{1} \times G_{2}$ (см. предложение 1 ниже). Однако эта подгруппа не будет, как легко понять, ни равномерной, ни квазиравномерной. Другими словами, плезиоравномерные подгруппы как новый объект появляются уже при столь естественной операции, как прямое произведение равномерных и квазиравномерных подгрупп.

В связи с понятием плезиоравномерности возникает немало естественных вопросов. Например, что произойдет, если в определении плезиоравномерности поменять местами равномерность и квазиравномерность подгрупп (т.е. предполагать, что подгрупша $P$ из этого определения квазиравномерна в $G$, а $H$ равномерна в $P)$ ? Не получится ли при этом новый класс подгрупп и соответствующих однородных пространств? Оказывается, что не получится (см. лемму ниже). Еще вопрос (навеянньй случаями компактных и квазикомпактных однородных пространств, где ответ на него положителен): если замкнутая подгруппа в $G$ содержит плезиоравномерную подгруппу $H$, то будет ли она сама плезиоравномерна? Есть немало других естественных вопросов, ответам на некоторые из них и посвящена данная статья. Основные результаты доказаны в теоремах 1 и 2 .

Некоторые результаты этой статьи были анонсированы еще десять лет назад в [6], но только недавно удалось выделить все основные свойства плезиоравномерных подгруп и оформить их в виде законченной статьи.

Одним из важных технических приемов при доказательстве результатов данной статьи является использование понятия оператора алгебраического замыкания на произвольной почти связной группе Ли. Поэтому начнем с изложения определения этого оператора и перечисления некоторых его основных свойств. Более подробное изложение можно найти, например, в [7], [8].

Вначале рассмотрим наиболее важный для нас частный случай односвязных групп Ли. Для произвольной связной и односвязной группы Ли $G$ в [7] построено (следуя идеям А.И. Мальцева, Л. Ауслендера и Г. Хохшильда-Дж. Мостова) алгебраическое расщелление. Так назьвается вложение $G$ в некоторую односвязную группу Ли $A(G)$ такую, что ее факторгруппа $A_{l}=A(G) / L(S)$ по линеаризатору $L(S)$ полупростой части (т.е. фактора Леви) $S$ группы Ли $G$ изоморфна связной компоненте единицы некоторой линейной алгебраической группы Ли, определенной над $\mathbb{R}$, точнее, ее группы вешественных точек. Напомним, что линеаризатором $L(F)$ связной группы Ли $F$ называется наименьший нормальньй делитель, факторгруппа по которому линейна (т.е. имеет точное линейное представление). Если группа Ли $F$ полупроста, то ее линеаризатор 
дискретен (и содержится в центре этой группы Ли, имея в нем конечный индекс). Для односвязной групшы Ли ее линеаризатор совпадает с линеаризатором ее полупростой части (подробнее см., например, [8]).

Следует отметить, что при построении алгебраического расщепления вместо связных групп Ли $G$ можно рассматривать и почти связные, т.е. имеющие только конечное число связных компонент. Для них можно повторить все этапы конструкции, приведенныев [7], и тоже получить конструкцию алгебраического расщепления. Далее, в [7] определен оператор $\delta$ алгебраического замыкания, действуюший на произвольных подмножествах $X$ в $A(G)$, а также порожденньй им оператор $\delta_{G}$.

Приведем конструкции этих операторов. Рассмотрим естественньй эпиморфизм $\pi$ : $A(G) \rightarrow A_{l}=A(G) / L(S)$ на линейную группу Ли, которая является связной компонентой единицы некоторой алгебраической группы $A$ (см. вьше). Пусть $\delta_{A}$ обозначает оператор алгебраического замыкания (относительно обычной топологии Зарисского) в $A$. Для произвольного подмножества $X$ в $A(G)$ рассмотрим $\delta_{A}(\pi(X)) \cap A_{l}$ - некоторое подмножество в $A_{l}$, имеющее конечное число связных компонент. Рассмотрим теперь подмножество $\pi^{-1}\left(\delta_{A}(\pi(X)) \cap A_{l}\right)$ и через $\delta(X)$ обозначим объединение тех связных (в евклидовой топологии) компонент этого подмножества, которые имеют непустое пересечение с $X$. Полученное подмножество $\delta(X)$ и назьвается алгебраическим замыканием подмножества $X$ в $A(G)$. Легко проверить, что оператор $\delta$ удовлетворяет всем аксиомам оператора топологического замыкания. Более того, его можно рассматривать как некоторое обобщение классического оператора алгебраического замькания в топологии Зарисского.

Если $X$-подгруппа в $A(G)$, то легко убедиться в том, что $\delta(X)=X \cdot \pi^{-1}\left(\delta_{A}(X) \cap A_{l}\right)_{0}$ (где ( ) 0 обозначает связную компоненту единицы). Положим, наконец, $\delta_{G}(X)=$ $\delta(X) \cap G$, где группа $G$ рассматривается как подгруппа в $A(G)$ (в силу упомянутого выше алгебраического расщепления). Перечислим некоторые свойства оператора $\delta_{G}$ (подробнее см. [7], [8]). Ясно, что $\delta_{G}(H) \supset H$ для любой подгруппы $H$. Далее, $\delta_{G}(H)$ нормализует $H$ и $H_{0}$. Наконец, $\delta_{G}(H)$ почти связна по модулю $L(S)$, т.е. $\delta_{G}(H) / \delta_{G}(H) \cap L(S)$ имеет только конечное число связных компонент (хотя сама подгруппа $\delta_{G}(H)$ может иметь и бесконечное число связных компонент, в отличие от случая классического алгебраического замыкания).

Первое рассматриваемое нами свойство плезиоравномерных подгрупा - транзитивность. Отметим, что для свойства компактности подобное свойство совершенно очевидно, для квазикомпактности оно тоже доказьвается без особого труда.

ТЕорема 1 (транзитивность плезиоравномерности). Пусть $G$ - некоторая группа Ли, ан и Предположим, что $H$ плезиоравномерна в $F$, a $F$ плезиоравномерна в $G$. Тогда $H$ плезиоравномерна в $G$.

ДоКАЗАТЕЛЬСТВо. Из определения плезиоравномерности следует, что существуют замкнутые равномерные подгруппы $P_{H} \subset F$ и $P_{F} \subset G$, которые содержат $H$ и $F$ соответственно в качестве квазиравномерных подгрупп. В результате имеем последовательность включений

$$
H \subset P_{H} \subset F \subset P_{F} \subset G \text {. }
$$

Ниже будет доказано, что $P_{H}$ плезиоравномерна в $P_{F}$. Предположим на минуту, что это уже сделано, т.е. существует такая замкнутая равномерная подгруппа $P \subset P_{F}$, ко- 
торая содержит $P_{H}$ в качестве квазиравномерной подгруппы. Так как $P_{F}$ равномерна в $G$, то $P$ тоже равномерна в $G$ (это очевидная транзитивность свойства равномерности). Далее, так как $H$ квазиравномерна в $P_{H}$, она будет квазиравномерна и в $P$ (это транзитивность свойства квазикомпактности; она вытекает из теоремы Фубини, доказательство можно найти в [9]). В результате получаем, что $P$ - равномерная подгруппа в $G$, содержащая $H$ в качестве квазиравномерной подгрупшы. В силу определения плезиоравномерности это и означает, что $H$ плезиоравномерна в $G$.

Итак, нам остается только доказать, что $P_{H}$ плезиоравномерна в $P_{F}$. Предварительно сделаем два упрощения.

Во-первых, мы можем считать, что группа Ли $G$ связна. Дело в том, что если $Q-$ некоторая плезиоравномерная подгруппа в произвольной групше Ли $G$, то $Q / Q \cap G_{0}-$ подгруппа конечного индекса в $G / G_{0}$ - группе связных компонент группы Ли $G$ (здесь $G_{0}$ - связная компонента единишы группы Ли $\left.G\right)$. Поэтому мы можем перейти, не нарушая справедливости предположений и утверждений теоремы, к рассмотрению подгруппы $Q \cap G_{0}$ в связной группе Ли $G_{0}$.

Во-вторьх, мы можем считать группу Ли $G$ односвязной, ибо если это не так, то переходим к универсальному накрытию и все подгрупшы в $G$ заменяем на их прообразы при этом накрытии. На свойствах равномерности, квазиравномерности и плезиоравномерности подгрупп такой переход не отразится.

Из сказанного следует, что доказательство теоремы 1 сводится к доказательству следующей леммы, которая представляет собой основной технический результат данной статьи.

Лемма. Пусть $G$ - связная, односвязная группа Ли, а $H$ и $F-$ две ее замкнутые подгруппь, причем $H$ содержится в $F$. Предположим, что $Н$ равномерна в $F$, а $F$ квазиравномерна в $G$. Тогда $H$ будет плезиоравномерна в $G$, причем в качестве подгруппь $P_{H}$, фигурирующей в определении плезиоравномерности для $H$, можно взять $\delta_{G}(H)$.

Это означает, что в определении плезиоравномерности понятия равномерности и квазиравномерности мохсно менять местами.

ДоказАтельСтво. Рассмотрим $N=N_{G}\left(F_{0}\right)$ - нормализатор в $G$ связной компоненты единицы $F_{0}$ подгрупшы $F$. Как доказано в [10], из квазиравномерности $F$ в $G$ вытекает, что этот нормализатор $N$ равномерен в $G$ (это утверждение можно назвать леммой Мостова-Вана). Поэтому мы можем использовать его в качестве $P_{F}$ в определении плезиоравномерности подгруппы $F$. Отсюда вытекает, что, заменив $G$ на $N$, мы можем считать $F_{0}$ нормальной подгруппой в $G$.

Пусть $\delta$-оператор алгебраического замыкания, а $A(G)$ - алгебраическая оболочка односвязной группы Ли $G$. Рассмотрим подгруппу $\delta_{G}(H)=\delta(H) \cap G$ и докажем, что она равномерна в $G$.

Положим $\Phi=\overline{F \cdot \delta_{G}(H)}$, где черта вверху обозначает обычное топологическое замыкание. Переходя к подгруппе конечного индекса в $F$ (что не влияет на справедливость утверждения леммы), мы можем считать, что $\delta_{G}(H) \cdot F_{0} \supset F$, и тогда будет $\Phi=\overline{\delta_{G}(H) \cdot F_{0}}$. Так как $\Phi \supset F$, то $\Phi$ тоже квазиравномерна в $G$ (это иногда назьвают наследственностью свойства квазикомпактности).

Подгруппа $\delta_{G}(H)$ почти связна по модулю $L(S)$ - линеаризатора полупростой части $S$ группы Ли $G$ (см. выше). С другой стороны, подгруппа $F_{0} \cdot \delta_{G}(H)$ имеет конечньй 
индекс в $F \cdot \delta_{G}(H)$. Это следует из того, что $\delta_{G}(H) \supset H$, а $F / H$ почти связно в силу равномерности $H$ в $F$. Но тогда из сказанного вьше получаем, что $\Phi$ почти связна по модулю $L(S)$. В силу леммы Мостова-Вана [9], примененной к $G / L(S)$, получаем, что $\Phi$ равномерна в $G$. Заменяя в случае необходимости $G$ на $\Phi$, мы можем считать, что $\Phi=G$, т.е. $F \cdot \delta_{G}(H)$ плотна в $G$ (в обычной топологии). Отсюда, в частности, следует, что $F_{0} \cdot\left(\delta_{G}(H)\right)_{0}$ нормальна в $G$. Так как группа Ли $G$ односвязна, любой нормальный делитель в ней обязательно замкнут. Следовательно, $F_{0} \cdot\left(\delta_{G}(H)\right)_{0}$ - замкнутьй нормальный делитель в $G$.

Подгруппа $F \cdot \delta_{G}(H)$ почти связна по модулю $L(S)$, поэтому она почти совпадает с $F_{0} \cdot\left(\delta_{G}(H)\right)_{0}$ по модулю $L(S)$. В силу этого из замкнутости $F_{0} \cdot\left(\delta_{G}(H)\right)_{0}$ в $G$ следует, что $\delta_{G}(H) \cdot F_{0}=G$.

Имеем следующие естественные отождествления пространств смежных классов (являюшиеся диффеоморфизмами гладких многообразий):

$$
G / \delta_{G}(H)=F_{0} \cdot \delta_{G}(H) / \delta_{G}(H)=F_{0} / F_{0} \cap \delta_{G}(H) .
$$

Но $\delta_{G}(H) \supset H$ и $H$ равномерна в $G$. Поэтому пространство $F_{0} / F_{0} \cap \delta_{G}(H)$ компактно. Отсюда вытекает, что $\delta_{G}(H)$ - равномерная подгруппа в $G$.

Докажем теперь, что $H$ квазиравномерна в $\delta_{G}(H)$. По условию $F$ квазиравномерна в $G$. Однородное пространство $G / F$ можно естественно отождествить с $F \cdot \delta_{G}(H) / F=$ $\delta_{G}(H) / F \cap \delta_{G}(H)$. Далее, имеем вложения $H \subset \delta_{G}(H) \cap F \subset \delta_{G}(H)$.

Рассмотрим однородное пространство $M=\delta_{G}(H) / H$. В силу указанного вьше свойства оператора $\delta_{G}$ подгруппа $H_{0}$ нормальна в $\delta_{G}(H)$. Положим $U=\delta_{G}(H) / H_{0}, \Gamma=$ $H / H_{0}$. Тогда $M=U / \Gamma$, где $\Gamma$ - дискретная подгруппа в $U$. На $U / \Gamma$ имеется, очевидно, естественная инвариантная мера. Поэтому на $M$ имеется инвариантная мера.

Подг руппа $H$ равномерна в $G$, поэтому $H$ равномерна и в $\delta_{G}(H) \cap F$. Далее, подгруппа $\delta_{G}(H) \cap F$ квазиравномерна в $\delta_{G}(H)$, ибо в силу равенства $\delta_{G}(H) \cdot F_{0}=G$ квазикомпактное однородное пространство $G / F$ естественным образом можно отождествить с $\delta_{G}(H) / F \cap \delta_{G}(H)$. Из сказанного вытекает, что инвариантная мера на $\delta_{G}(H) / H$ конечна, т.е. $H$ квазиравномерна в $\delta_{G}(H)$. Тем самым, подгруппа $H$ является плезиоравномерной в $G$, причем в качестве $P_{H}$ в определении плезиоравномерности можно взять подг руппу $\delta_{G}(H)$. Лемма доказана.

Как уже было показано выше, утверждение теоремы 1 вытекает из доказанной леммы.

Из теоремы 1 непосредственно вытекает следствие, котороепредставляет собой транзитивность свойства плезиоравномерности в максимально широкой форме. Его можно также назвать свойством сокрашения цепочек подгрупш.

СЛЕДСТВИЕ. Пусть для некоторой группь Ли $G$ имеется иепочка замкнутых noдәpynn

$$
H=F_{0} \subset F_{1} \subset F_{2} \subset \cdots \subset F_{k-1} \subset F_{k}=G,
$$

причем каждая из подгрупп $F_{i}$ равномерна или квазиравномерна в $F_{i+1}$. Тогда подгруппа $H$ плезиоравномерна в $G$.

Следующая теорема показьвает, что свойство плезиоравномерности обладает наследственностью (справедливость которой для свойств равномерности и квазиравномерности хорошо известна). 
ТЕОремА 2. Пусть $G$ - некоторая группа Ли, а $H$ - плезиоравномерная подгруппа в $G$. Тогда любая замкнутая подгруппа $F$ в $G$, содерәсащая $H$, тохе будет плезиоравномерной в $G$.

ДокАЗАтЕльство. Пусть $P_{H}$ - та подгруппа в $G$, которая в силу определения плезиоравномерности подгрупш $H$ является равномерной подгрупой в $G$, содержащей $H$ в качестве квазиравномерной подгрупшы. Как видно из доказательства теоремы 1 , в качестве такой $P_{H}$ можно взять $\delta_{G}(H)$.

Рассмотрим подгрупу $\delta_{G}(F)$. Она содержит $\delta_{G}(H)$, а так как последняя равномерна в $G$, то и $\delta_{G}(F)$ тоже равномерна в $G$ (мы использовали очевидную наследственность свойства равномерности).

Докажем теперь, что $F$ квазиравномерна в $\delta_{G}(F)$. Рассмотрим два семейства включений:

$$
H \subset F \subset \delta_{G}(F) \subset G, \quad H \subset \delta_{G}(H) \subset \delta_{G}(F),
$$

в которых $H$ квазиравномерна в $\delta_{G}(H), \delta_{G}(H)$ равномерна в $\delta_{G}(F)$, а та, в свою очередь, равномерна в $G$. Заменяя $G$ на $\delta_{G}(F)$, мы можем при доказательстве теоремы 2 считать, что $\delta_{G}(F)=G$. Но тогда, в частности, $F_{0}$ будет нормальна в $G$. Докажем, что $F$ квазиравномерна в $G$.

На однородном пространстве $G / F$ существует инвариантная мера. Это утверждение доказьвается точно так же, как при доказательстве леммы вьше, и основьвается на том, что $F_{0}$ нормальна в $G$. Остается доказать, что эта мера конечна.

Рассмотрим $\overline{\delta_{G}(H) \cdot F_{0}}$; эта подгруппа почти содержит $F$ (т.е. ее пересечение с $F$ имеет в $F$ конечньй индекс). Имеем последовательность включений

$$
H \subset F \subset \delta_{G}(H) \cdot F_{0}=U \subset G .
$$

Подгруппа $U$, определенная вьше, равномерна в $G$. Снова, как и при доказательстве леммы, мы можем, заменяя в случае необходимости $G$ на $U$, считать, что $U=G$. Из этого равенства так же, как при доказательстве леммы, выводится, что $\delta_{G}(H) \cdot F_{0}=G$.

Рассмотрим однородное пространство $G / F$. Его можно естественньп образом отождествить с $\delta_{G}(H) \cdot F / F=\delta_{G}(H) / F \cap \delta_{G}(H)$. Из условия доказьваемой теоремы вытекает, что $H$ квазиравномерна в $\delta_{G}(H)$. Так как $H$ содержится в $F \cap \delta_{G}(H)$, то на однородном пространстве $\delta_{G}(H) / F \cap \delta_{G}(H)$ существует конечная мера. Поэтому и инвариантная мера на $G / F$ тоже конечна. Этим доказано, что $F$ квазиравномерна в $G$ (при условии, что $\delta_{G}(F)=G$ ) и потому плезиоравномерна в $G$.

Еще одно свойство, которое интересно рассмотреть для плезиоравномерных подгруп, - это мультипликативность, которую можно понимать несколькими разными способами.

ПРЕДЛОЖЕНИЕ 1. Пусть $H_{1}, H_{2}$ - плезиоравномерные подгруппы в группах Ли $G_{1}, G_{2}$ соответственно. Тогда $H_{1} \times H_{2}$ - плезиоравномерная подгруппа в $G_{1} \times G_{2}$.

ДокАЗАТЕЛЬСтво. Рассмотрим следующую цепочку подгрупш в $G_{1} \times G_{2}$ :

$$
H=H_{1} \times H_{2} \subset F=H_{1} \times G_{1} \subset G=G_{1} \times G_{2} .
$$

Ясно, что $H$ плезиоравномерна в $F$, а $F$ плезиоравномерна в $G$. Но тогда из теоремы 1 следует, что $H=H_{1} \times H_{2}$ плезиоравномерна в $G=G_{1} \times G_{2}$. 
Мультипликативность в таком понимании имеет место, очевидно, и для свойств равномерности и квазиравномерности. Другое понимание мультипликативности можно сформулировать так: если $H_{1}, H_{2}$ - две замкнутые плезиоравномерные подгруппы в $G$, то $\left\langle H_{1} \cdot H_{2}\right\rangle$ - замкнутая подгруппа, порожденная их произведением, - тоже плезиоравномерна. Но в такой формулировке свойство мультипликативности очевидно и вытекает из наследственности, так как $\left\langle H_{1} \cdot H_{2}\right\rangle$ содержит плезиоравномерную подгрупу $H_{1}$ (или $H_{2}$ ) группы Ли $G$.

В заключение рассмотрим один отрицательный результат. Речь идет об ответе на следуюший естественньй вопрос: во всякой ли группе Ли $G$ имеется нетривиальная плезиоравномерная подгруппа $H$ ? При этом, конечно, нужно ограничиться случаем локально эффективных подгрупп, т.е. таких, которые не содержат нетривиальных связных нормальных делителей групшы Ли $G$ (в противном случае, например, в любой разрешимой группе Ли легко построить плезиоравномерную подгруппу). Оказьвается, что даже для разрешимых групा Ли ответ на этот вопрос отрицательный и контрпример доставляет простейшая неабелева разрешимая групша Ли $R_{2}$. Эту групу Ли можно рассматривать как связную компоненту единицы группы аффинных преобразований прямой. Групша $R_{2}$ может быть представлена в виде полупрямого произведения $\mathbb{R} \cdot \mathbb{R}$, множители которого можно рассматривать соответственно как группы переносов и растяжений аффинной прямой. Оказьвается, что в этой группе Ли нет нетривиальных локально эффективных плезиоравномерных подгрупп.

Вначале покажем, что в разрешимой групе Ли понятия равномерности, квазиравномерности и плезиоравномерностии эквивалентны.

ПРЕДЛОЖЕНИЕ 2. Пусть $G$ - разрешимая группа Ли, а $H$ - замкнутая подгруппа в $G$. Тогда следующие утверждения әквивалентны:

i) подгруппа $Н$ равномерна в $G$;

ii) подгруппа $H$ квазиравномерна в $G$;

iii) подгруппа $H$ плезиоравномерна в $G$.

ДокАЗАТЕЛЬСтво. Эквивалентность утверждений і) и іi) доказана в [5]. По определению плезиоравномерности и из i) и из іi) вытекает іiі). Докажем, что из іiі) вытекает i).

Пусть $H$ плезиоравномерна в $G$. Тогда существует такая замкнутая равномерная подгруппа $P$ в $G$, которая содержит $H$ в качестве квазиравномерной подгрупшы. Но тогда в силу импликации іi) $\Longrightarrow$ i) получаем, что $H$ равномерна в $F$, апотому равномерна и в $G$.

Докажем теперь, что в $R_{2}$ нет нетривиальных локально эффективных плезиоравномерных подгрупп. Предположим обратное и пусть $H$ - одна из таких подгрупп. Так как $\operatorname{dim} R_{2}=2$, то $\operatorname{dim} H=0$ или 1.

Если $\operatorname{dim} H=0$, то $H$ - дискретная подгруппа. Из ее плезиоравномерности в силу предложения 2 следует, что она и равномерна, а потому содержащая ее группа Ли должна быть унимодулярна (см. [8]). Но группа Ли $R_{2}$ не унимодулярна. Получили противоречие.

Пусть теперь $\operatorname{dim} H=1$. Рассмотрим нильрадикал $N$ групшы Ли $R_{2}$; он одномерен. Для равномерных подгрупп разрешимых групп Ли известно, что их связная компонента единицы содержится в $N$ [11]. Плезиоравномерная подгруппа в разрешимой группе Ли равномерна в силу предложения 2. Поэтому должно быть $H_{0} \subset N$. Но $\operatorname{dim} H_{0}=1=\operatorname{dim} N$ и потому $H_{0}=N$. Однако $N-$ нормальньй делитель в $R_{2}$, и 
мы снова получаем противоречие с предположением о локальной эффективности подгруппы $H$. Итак, во всех возможных случаях предположение о существовании в $R_{2}$ нетривиальной плезиоравномерной локально эффективной подгрупшы приводит к противоречию.

\section{СПИСОК ЦИТИРОВАННОЙ ЛИТЕРАТУРЫ}

[1] Горбацевич В.В. О плезиокомпактных однородных пространствах I // Сиб. матем. ж. 1989. T. 30. № 2. C. $61-72$.

[2] Горбацевич В. В., Онищик А. Л.Группы Ли преобразований // Итоги науки и техники. Совр. пробл. матем. Фундам. направления. М.: ВИНИТИ, 1988. С. 103-240.

[3] Горбацевич В. В. О плезиокомпактных однородных пространствах II // Сиб. матем. ж. 1991. T. 32. № 2. C. $13-25$.

[4] Горбацевич В. В. РасслоениеЗейферта для плезиокомпактного однородного пространства // Сиб. матем. ж. 1996. Т. 37. № 2. С. 301-313.

[5] Mostow G. Factor spaces of solvable groups // Ann. Math. 1954. V. 60. № 1. P. 1-27.

[6] Горбацевич В.В. О некоторых классах однородных пространств, близких к компактным // Докл. АН СССР. 1988. Т. 303. № 4. С. 785-788.

[7] Горбацевич В.В. Расщепление групп Ли и его применения к изучению однородных пространств // Изв. АН СССР. Сер. матем. 1979. Т. 43. №6. С. 1227-1258.

[8] Винберг Э. Б., Горбацевич В.В., Онищик А. Л. Строение групп и алгебр Ли // Итоги науки и техники. Совр. пробл. матем. Фундам. направления. Т. 41. М.: ВИНИТИ, 1990. C. 5-258.

[9] Рагунатан М. Дискретные подгруппы групп Ли. М.: Мир, 1977.

[10] Wang S. P. Homogeneous spaces with finite invariant measure // Amer. J. Math. 1973. V. 98. № 2. P. 311-324.

[11] Auslander L. An exposition of the structure of solvmanifolds // Bull. Amer. Math. Soc. 1973. V. 79. № 2. P. 227-261. 